\begin{tabular}{|c|c|c|c|}
\hline Eiszeitalter u. Gegenwart & 38 & $37-51$ & Hannover 1988 \\
& & $8 \mathrm{Abb} ., 1 \mathrm{Tab}$. & \\
\hline
\end{tabular}

\title{
Vegetationsentwicklung, Paläoböden, Seespiegelschwankungen: Untersuchungen an eem- und weichselzeitlichen Sedimenten vom Südrand des Harzes
}

\author{
Werner Ricken \& Eberhard GrÜGer *)
}

\begin{abstract}
Collapse sink, Eemian, Würm, extinct lake, lake sediment, sand, silt, mud, peat, $\mathrm{C} / \mathrm{N}$ ratio, pollen diagram, vegetation, paleosol, soil profile, loess, lake level change

Lower Saxony, Northern German Hills, Harz Mountains Southern Border
\end{abstract}

Kurzfassung: Die jungpleistozäne Sedimentserie des Aschenhütter Erdfalls (südlicher Harzrand, Niedersachsen) bietet die interessante Möglichkeit, palynologische und geologische Befunde miteinander zu verknüpfen. Die Sedimentserie besteht aus limnischen und telmatischen Ablagerungen und Lößderivaten mit eem- und weichselzeitlichen Paläoböden. Die limnisch-telmatischen Sedimente umfassen nach dem pollenanalytischen Befund die Hainbuchenzeit und die darauffolgenden Abschnitte des Eem-Interglazials, das Brörup-Interstadial (sensu ANDERSEN) und Teile der diesem vorausgehenden bzw. folgenden Stadiale (Herningbzw. Rederstall-Stadial).

Anders als an den meisten eemzeitlichen Untersuchungspunkten, die fast alle in den ehemals vereisten Tieflandsgebieten liegen, war die Fichte am Harzrand schon während der Hainbuchenzeit eine der wichtigen Baumarten. Die interstadiale Vegetationsentwicklung läßt keinen Klimarückschlag erkennen, wie er in Gegenden mit einer thermisch anspruchsvolleren Vegetation oder nahe an den Verbreitungsgrenzen der einzelnen Baumarten im Brörup-Interstadial gewöhnlich deutlich wird. Die botanischen Untersuchungen ergaben auch keine Hinweise auf ein Vorkommen von Bruckenthalia und Picea omoricoides bei Aschenhütte.

Die pollenanalytisch datierte limnisch-telmatische Sedimentfolge verzahnt sich mit Lößpaläoböden und ermöglicht deren stratigraphische Einordnung. Die pollenführenden Sedimente gehen im Uferbereich in den Eem-Boden und in frühweichselzeitliche Bleichhorizonte und Fließerden über; im Hangenden treten Lößböden des Stillfried-B-Interstadials auf (Hattorfer und Lohner Boden der mittleren Weichselzeit).

*) Anschriften der Autoren: Dr. W. RickeN, GeologischPaläontologisches Institut der Universität Tübingen, Sigwartstraße 10, D - 7400 Tübingen. - Prof. Dr. E. GRÜGER, Institut für Palynologie und Quartärwissenschaften der Universität Göttingen, Wilhelm-Weber-Straße 2, D - 3400 Göttingen.
Die Untersuchungen zur Faziesverteilung der Sedimente und zum Auftreten von Wasserpflanzen erlauben Aussagen über Spiegelschwankungen des Aschenhütter Sees. Spiegelhöchststände wurden nach dem Ende des Eem-Interglazials und während des Brörup-Interstadials erreicht. Dagegen sank der Wasserspiegel im Verlauf des Herning-Stadials, und im Rederstall-Stadial verlandete der Aschenhütter See. Die Spiegelschwankungen werden mit Niederschlags- und Verdunstungsänderungen als Folge von Klimaschwankungen erklärt.

[Vegetational Development, Paleosoils and Lake Level Changes: Studies on Eemian and Weichselian Sediments from the Southern Borders of the Harz Mountains]

Abstract: The Upper Pleistocene sediments of the Aschenhütte sink-hole (west of Herzberg am Harz, Lower Saxony, West-Germany) enable one to make interesting correlations between palynological and geological results. The sequence is composed of limnic-telmatic deposits (Eemian to Lower Weichselian) and loess with paleosoils (Weichselian). Sedimentation started during the hornbeam-dominated phase of the Eemian interglacial period and continued throughout the Eemian, the Weichselian Brörup interstadial (sensu Andersen) and parts of the preceding and the following stadial phases, the Herning and the Rederstall stadials.

As opposed to most of the known Eemian sites spruce was a major tree species during the hornbeam-dominated phase of the Eemian. The vegetational development during the interstadial phase does not show a period of climatic deterioration as is the case for the Brörup interstadial when considering regions with a more demanding vegetation or regions close to the natural boundaries of the tree species concerned. Pollen or seeds of Bruckenthalia and Picea omoricoides have not been found in the Aschenhütte cores.

The limnic-telmatic sediments interlock with loesspaleosoils (Eemian soil and Lower Weichselian bleaching soils) at the lake shore. They are overlaid by loess paleosoils 
of the Stillfried-B interstadial (Hattorf Soil and Lohne Soil). Lake level fluctuations were determined by means of the facies distribution and isochrones as defined by pollen analy. sis. A relatively high stand of the lake level existed after the end of the Eemian interglacial and during the Brörup interstadial periods. In the course of the Herning stadial period the water level dropped, whereas during the Rederstall stadial period the lake basin was covered by sediments and therefore dried up.

\section{Einleitung}

Der jungpleistozäne Erdfall von Aschenhütte am Harz (hier auch Aschenhütter See genannt) enthält eine für die Pleistozänstratigraphie bedeutsame Schichtenfolge, welche - nicht ganz lückenlos - den Zeitabschnitt vom ausgehenden Eem-Interglazial bis in das Hochglazial der Weichselzeit umfaßt. Die Abfolge dokumentiert die Verfüllung eines kleinen, im EemInterglazial entstandenen Erdfallbeckens mit Mudden, Torfen und Lößderivaten. Die Untersuchungen galten 1. der Vegetationsentwicklung und pollenanalytischen Datierung der limnischen und telmatischen Sedimente, 2. der Verzahnung und Überlagerung von Lößpaläoböden mit den datierten limnischtelmatischen Sedimenten und 3. den Seespiegelschwankungen in Abhängigkeit von Warm- und Kaltphasen.

Der ehemalige Aschenhütter See (R 3590375 H 5728650, $240 \mathrm{~m} \mathrm{NN}$ ) liegt unmittelbar am Südrand des Harzes bei Aschenhütte, Kreis Osterode

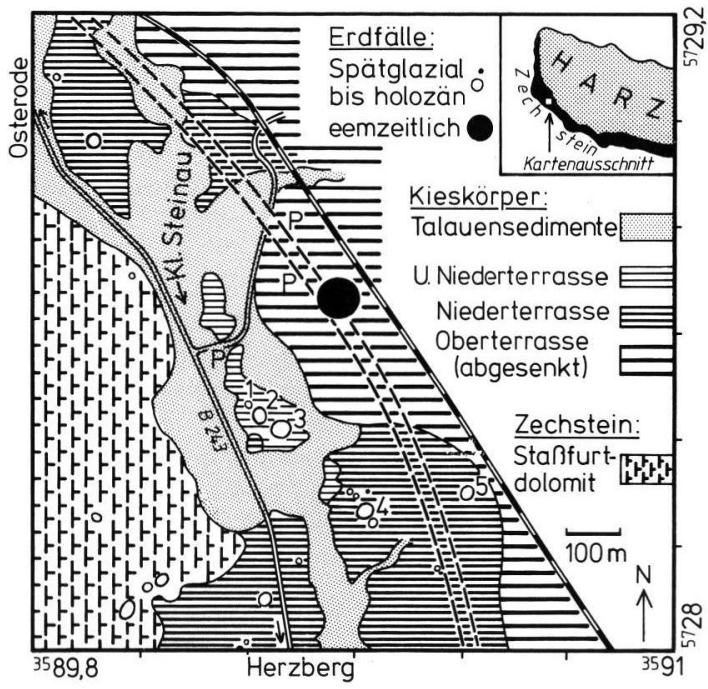

Abb. 1: Untersuchungsgebiet und Lage des ehemaligen Aschenhütter Sees (schwarzer Punkt).

Fig. 1: Study area showing location of former Lake Aschenhütte (Eemian sink-hole, black circle).
(Niedersachsen) im Nordwestteil einer ausgedehnten, mit quartären Flußkiesen gefüllten Subrosionssenke des Werraanhydrits (Zechstein, Abb. 1). Der Erdfallsee hat sich auf vermutlich elsterzeitlichen Kiesen der Oberterrasse gebildet, welche noch vor dem Erdfalleinbruch subrosiv abgesenkt worden sind, so daß die Kiese eine nach SW geneigte Fläche bilden (JORDAN 1976; RICKEN 1982). Durch das Einsinken des mehr als $60 \mathrm{~m}$ breiten Erdfalltrichters in die geneigte Kiesfläche entstanden unterschiedlich hohe Böschungen, die im NE etwa $15 \mathrm{~m}$, im SW aber nur $6 \mathrm{~m}$ Höhe erreichten. Dadurch konnte sich später im SW ein Überlauf für den See bilden und - nach seiner Verlandung - die verbliebene Restsenke mit Lößderivaten vollständig verfüllt werden. Die Sedimentfüllung des Aschenhütter Erdfalls wurde 1975 unerwartet beim Anlegen eines Straßeneinschnitts (neue Trasse der B 243) angefahren und etwa bis zur Hälfte ausgeräumt. Wie Sondierbohrungen zeigten, reichen die Seesedimente noch mehr als $50 \mathrm{~m}$ von der östlichen Straßenböschung nach NE. Sie erreichen aber nicht den parallel zur Straße liegenden Bahneinschnitt (Abb. 1).

\section{Methoden}

Für die Untersuchungen standen am Aufschluß entnommene Proben (Nr. 30-32) des Profils H 75, Abb. 2 und 4) und zahlreiche mit einem EdelmanBohrer (Eijkelkamp-Bohrer) oder einer Schlitzsonde gewonnene Proben zur Verfügung. Das Profil H 77 (Abb. 2 und 4) wurde pollenanalytisch genau, zehn weitere Profile und mehrere aus dem Abraum geborgene Torfblöcke übersichtsweise pollenanalytisch untersucht, so daß die Faziesverteilung und Isochronen rekonstruiert werden konnten. Die Pollenproben wurden den der Bohrkrone des Edelman-Bohrers seitlich anhängenden Teilkernen $(2 \times 2 \times 5 \mathrm{~cm})$ entnommen. Die Aufbereitung der Proben erfolgte in der üblichen Weise (HF, KOH, Azetolyse). Die Pollendichte war in allen Proben hoch, die Pollenerhaltung im allgemeinen befriedigend. Für die Untersuchung der größeren Pflanzenreste wurde das restliche Probenmaterial (bis zu $400 \mathrm{ml}$ je Probe) in $10 \%$ iger $\mathrm{KOH}$ eingeweicht und dann unter fließendem Wasser gesiebt (Fig. 4b links). Die Kohlenstoffund Stickstoffbestimmungen wurden gasanalytisch im Institut für Bodenwissenschaften der Universität Göttingen durchgeführt.

\section{Stratigraphie}

Drei Sedimentkomplexe können unterschieden werden (Abb. 2): 
ISOCHRONEN

- GESICHERT ---VERMUTET

DIAGRAMMABSCHNITTE

$\begin{array}{clcl}5 & \text { SEGGENHORIZONT } & 5 & \text { STADIAL 1 (MIT UMGELAGERTEM } \\ 10-11 & \text { STADIAL 2 } & & \text { EEM-MATERIAL) } \\ 6 \cdot 9 & \text { FW-INTERSTADIAL } & 1-4 & \text { EEM-INTERGLAZIAL }\end{array}$

$\mathrm{NW}$ FLIESSERDEN
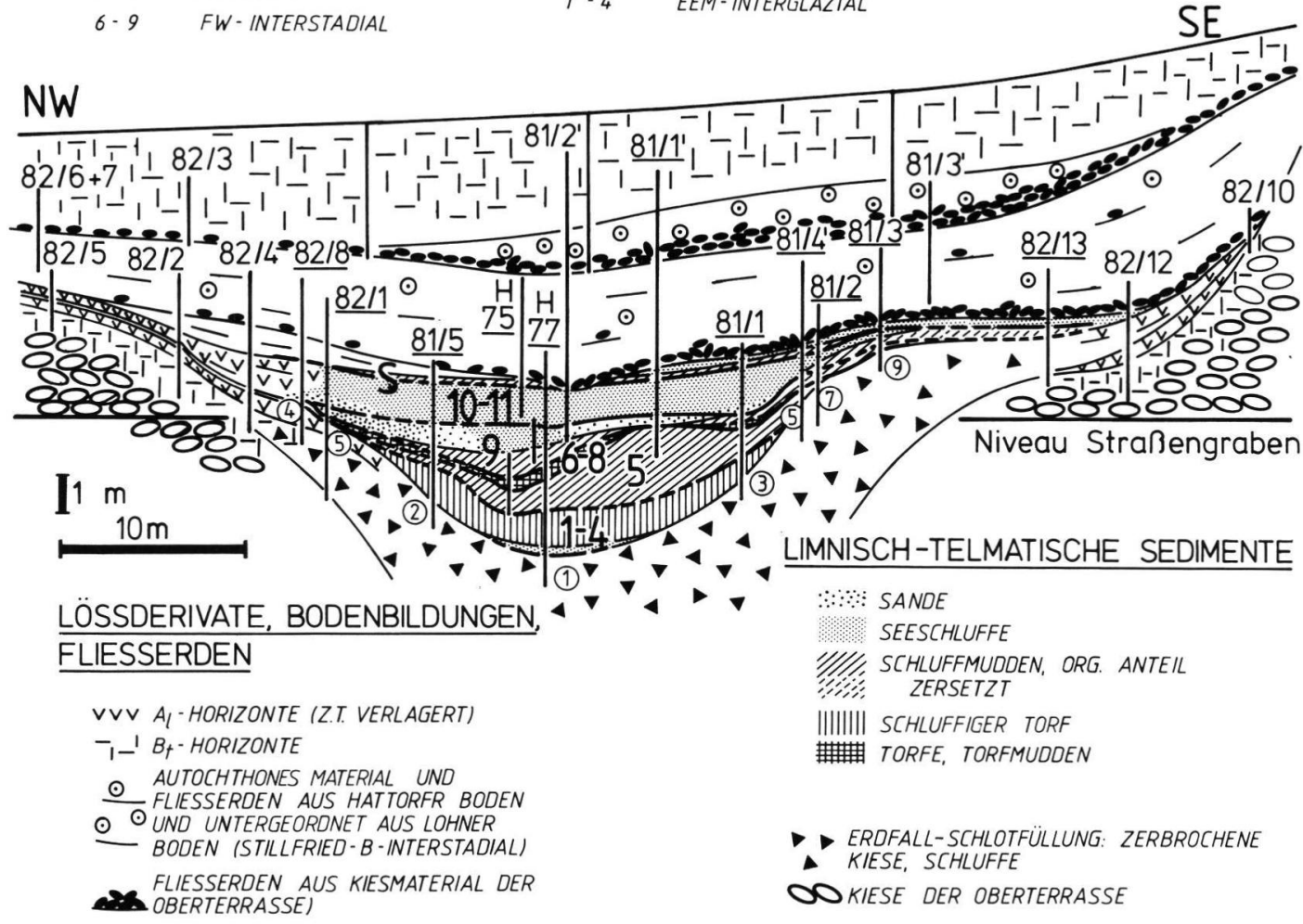
EEM-MATERIAL)

INTERGLAZIAL

Abb. 2: Sedimente und Isochronen des Aschenhütter Erdfalls entlang der nordöstlichen Einschnittsböschung der Straße Osterode-Herzberg. 82/2: Bohrung; 81/5: Bohrung oder Aufschluß pollenanalytisch untersucht.

Die übrigen Zahlen sind die Nummern der Abschnitte (DA) des Pollendiagramms (Abb. 4).

Fig. 2: Distribution of sediments and isochrones in the Aschenhütte sink-hole. Cross section along the northeastern road cut of the motorway Osterode-Herzberg. 82/2: drilling site, 81/5: drilling site or outcrop with palynological investigation.

The other numbers are the numbers of the pollen zones (DA) of the pollendiagram (Fig. 4).

1. Im unteren Teil der Erdfallsenke befinden sich bis $4,5 \mathrm{~m}$ mächtige limnische und telmatische Sedimente (Eem bis Frühweichsel), deren Obergrenze wegen der Setzung der Torfe im Profil nach unten durchgebogen ist.

2. Mit den limnisch-telmatischen Sedimenten verzahnen sich Paläoböden, Fließerden und Sande (Abb. 2), die pollenanalytisch mit den Seesedimenten parallelisiert werden können.

3. Die unter 1 und 2 genannten Sedimente werden von mittel- bis jungpleistozänen Lößderivaten von maximal $8 \mathrm{~m}$ Mächtigkeit überlagert. Die Lößderivate enthalten die Paläoböden des Stillfried-B-Interstadials.
Die durchweg karbonatfreien limnisch-telmatischen Sedimente des Erdfalles lassen sich wie folgt gliedern und datieren (Nomenklatur im wesentlichen nach MerkT et al. 1971, DA = Abschnitt des Pollendiagramms Abb. 4):

DA 1-4: Eem-Interglazial. Über das kiesige Füllmaterial des Erdfallschlotes wurden anfangs Schluffe (DA 1), dann schluffiger Torf mit organischen Kohlenstoffgehalten bis zu $34 \%$ sedimentiert.

DA 5: Frühweichsel-Stadial 1. Es kam zur Ablagerung von teilweise geschichteten Schluffmudden mit kontinuierlich von 15 auf $8 \%$ abnehmenden Kohlenstoffgehalten. Dieser Befund wird mit Resedimentation von eemzeitlichen Sedimenten erklärt (vergl. Kap. 4 und 8). 
DA 6-9: Frühweichsel-Interstadial. Nach einer pollenanalytisch erfaßbaren Schichtlücke, welche vermutlich mit einem kurzfristigen Trockenfallen des Aschenhütter Sees zusammenhängt, wurden in der Frühphase des Interstadials (DA6 bis Anfang 9) holzreiche Torfe, im mittleren und ausgehenden Interstadial aber limnische Sedimente mit kontinuierlich abnehmenden Anteilen an organischer Substanz abgelagert (Abb. 3). Dabei wurden im Bereich der Seemitte feingeschichtete Schluffmudden, Seeschluffe und stellenweise auch Sande sedimentiert.

DA 10-11: Frühweichsel-Stadial 2. Die Sedimentation kohlenstoffarmer, feingeschichteter Seeschluffe des ausgehenden Interstadials geht ohne Unterbrechung in das Stadial 2 über. Besonders in den Sedimenten des Abschnitts 11 fielen faserige Pflanzenreste auf, die wahrscheinlich von Seggen und Gräsern stammen: Die Verflachung des Sees, die sich damit

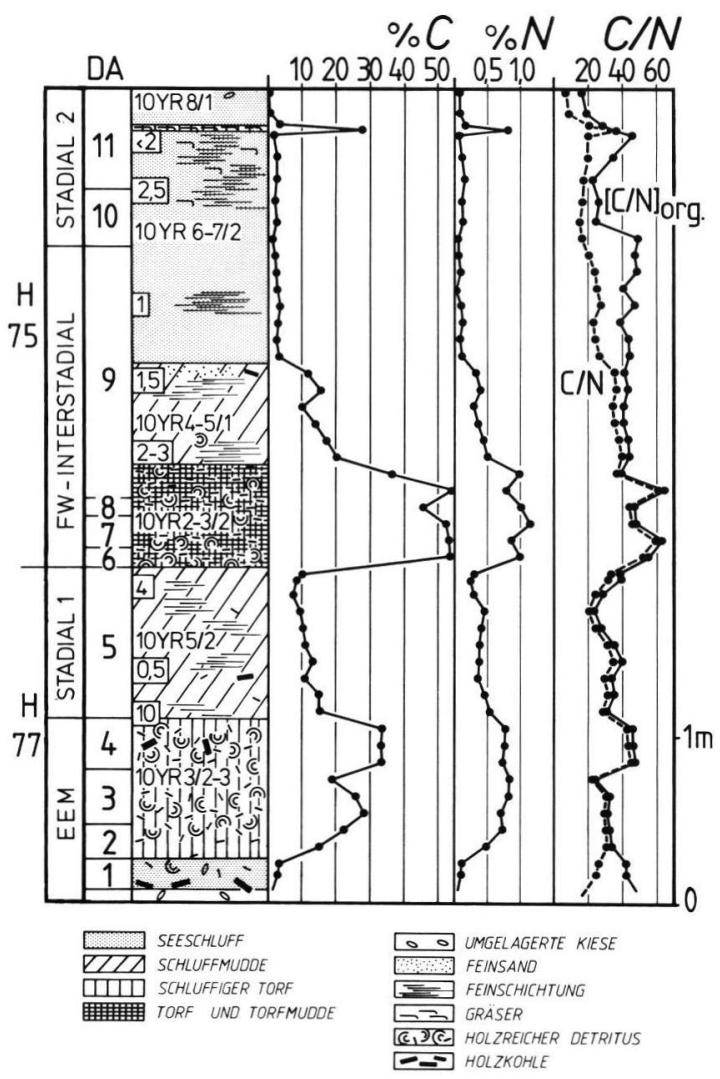

Abb. 3: Verteilung der limnischen und telmatischen Sedimente sowie Kohlenstoff- und Stickstoffgehalte der karbonatfreien Proben für den Bereich der Seemitte in den Profilen $\mathrm{H} 75$ und $\mathrm{H} 77$.

Fig. 3: Carbonate-free limnic and telmatic sediments and their carbon and nitrogen contents in the sections $\mathrm{H} 75$ and $\mathrm{H} 77$ from the middle of the former lake. andeutet, führte zur Bildung eines vermutlich isochronen, $5 \mathrm{~cm}$ mächtigen Verlandungshorizontes mit besonders vielen Gräsern und Seggen, die - bei gleichzeitiger Waldlosigkeit - einen Anstieg des Kohlenstoffgehalts im Sediment von 2 auf $28 \%$ verursachten ( = „Seggenhorizont”, Abb. 2 und 3).

\section{Ergebnisse der Pollen- und Großrestanalysen}

Zwei Waldphasen konnten nachgewiesen werden. Die ältere davon (DA 1-4) kann wegen des zeitweise reichen Vorkommens von Carpinus, Corylus, Picea, Abies u. a. thermisch relativ anspruchsvollen Baumarten einem Interglazial zugeordnet werden. In der jüngeren Waldzeit (DA 7-9) waren nie andere Baumarten als Birke, Kiefer und Fichte von Bedeutung. Die jüngere Waldphase ist demnach einem Interstadial zuzuordnen (vergl. Kap. 5).

Das Profil $\mathrm{H} 77$ ist an mehreren Stellen gestört (Abb. 2 und 4): zwischen DA 1 und 2 sowie DA 5 und 6 sind Schichtlücken nachgewiesen; eine weitere könnte an der Grenze DA 3a/3b liegen.

DA 1 (484-505 cm): Übergang von einer Eichenmischwaldzeit mit Taxus zu einer Zeit der CarpinusVorherrschaft (Quercus 13,1-5,2\%, Corylus $162-103,5 \%$, Taxus 1,6-0,2\%, Carpinus 3,8-18\%, Picea um 50\%, Alnus 59-137\%).

DA 2 (464-484 cm): Beginn nach einer Schichtlücke, die durch einen unvermittelten Wechsel von einer schluffig-tonigen, stark humosen Mudde (DA 1) zu schluffigem Torf (DA 2) gekennzeichnet ist. Der DA 2 unterscheidet sich pollenfloristisch kaum vom DA 1. Rhamnus frangula, Abies und Osmunda (bis 1,6\%, 1,2 resp. $4,4 \%$ ) treten neu auf. Die stark schattende Hainbuche verdrängte die lichtbedürftigeren Baumarten und gelangte zur Vorherrschaft (55\%). Die Fichte kam zu dieser Zeit bereits in der unmittelbaren Umgebung des Aschenhütter Erdfalls vor (Holzfunde).

DA 3 (428-464 cm): Kennzeichnend sind Fichtendominanz, abnehmende Carpinus-Werte und hohe Abies- und Osmunda-Anteile (max. 13,5 resp. $34 \%$ ). Ulmus, Tilia, Fraxinus, Acer und Corylus, aber auch Taxus, Hedera, Ilex und Viscum sind (und bleiben von hier an) völlig bedeutungslos. Die Kurven von Betula und Pinus beginnen jedoch anzusteigen. Vorübergehend kam es zu einer Ausbreitung der Tanne. Hainbuche, Fichte und Tanne bildeten die Wälder der Umgebung, in denen an geeigneten Standorten vermutlich auch Quercus noch vorkam.

DA 4 (395- $428 \mathrm{~cm}$ ): Weiterer Rückgang der Anteile der Laubholzarten außer der Birke, deren Werte 
ansteigen. Pinus erreicht ihren Höchstwert (33\%). Auch die Anteile der krautigen Arten ( = NBP) - vor allem die Anteile der Gramineae und die SphagnumWerte - nehmen stark zu. Die Calluna-Kurve erreicht $2 \%$, und die geschlossene Artemisia-Kurve beginnt.

In diesen Befunden spiegeln sich Veränderungen der Vegetation wider, wie sie für das Ende von Waldzeiten seit langem bekannt sind: Anspruchsvollere Baumarten verschwinden allmählich, und es kommt zur Bildung von Kiefernwäldern. Die in den schluffigen Torfen der DA 2-4 enthaltenen Samen und Früchte sowie einige Pollenkurven geben ergänzend Auskunft über die Art der Vegetation im Bereich der Erdfallsenke selbst. Hier wuchsen damals - wie es für nasse Waldlichtungen typisch ist - Schwarzer Holunder, zeitweise auch Weiden, Himbeeren, mehrere Hahnenfußarten, Seggen und Torfmoos. Holz resp. Nadeln beweisen das Vorkommen von Erlen und Fichten. Aus der Sedimentverbreitung (Abb. 2) kann geschlossen werden, daß der sumpfige Bereich innerhalb der Erdfallsenke nur einen Durchmesser von 5-15 m hatte. Ein See bildete sich erst zur Zeit des DA 5, wie die zunächst spärlichen, dann aber zahlreichen Nachweise von Potamogeton (Bestimmungen nach AALTO 1970) und Batrachium belegen.

DA 5 (316-395 cm): Dieser Diagrammabschnitt unterscheidet sich vom DA 4 durch noch höhere NBPund Betula-Werte (28-37\% bzw. 17-23\%), niedrigere Pinus- und Picea-Anteile und den Beginn der Juniperus-Kurve. In keinem der älteren Abschnitte ist die Zahl der nachgewiesenen NBP-Taxa so groß wie im DA 5. Darunter befinden sich solche, deren Vorkommen gewöhnlich als Hinweis auf offene Standorte und stadiale Klimabedingungen angesehen wird wie Artemisia, Ephedra, Gypsophila, Chenopodiaceae, Polygonum bistorta-Typ, Sanguisorba officinalis, Thalictrum u. a. Daneben sind anspruchsvollere Baumarten regelmäßig, z. T. reichlich nachgewiesen wie Quercus (bis 2,1\%), Abies (bis 1,9\%), Carpinus (bis 9,3\%), Corylus (bis 6,8\%) und Alnus (bis 37\%). Aber auch Einzelnachweise von Hedera-, Viscumund Ilex-Pollen sind verzeichnet. Dieses Phänomen kann mit partieller Resedimentation von eemzeitlichen Sedimenten (DA 1-4) erklärt werden. Als autochthon kann der Pollen der während der Zeit der Klimaverschlechterung (DA 4 und 5) neu auftretenden Taxa gelten. Sie zeigen die Verarmung und Auflichtung der Wälder wahrscheinlich bis zur Ausbildung einer offenen Vegetation mit lichten Birkenbeständen an. Der Pollen anderer Taxa, vor allem derjenige der anspruchsvolleren Waldbäume, stammt wahrscheinlich - wie auch ein Teil der Großreste aus den interglazialen Sedimenten, die durch Wellenbewegung bei wechselnder Wassertiefe erodiert und danach in dem kleinen Seebecken erneut sedimentiert werden konnten (vergl. Kap. 8). Das Ende des Interglazials dürfte an der Grenze DA 4/5 zu suchen sein.

Der DAs endet an einer pollenanalytisch nachgewiesenen Schichtlücke, die auch am abrupten Wechsel von Schluffmudde zu Torf erkennbar ist. Die Torfbildung hielt bis in die Zeit des DA9 an.

DA 6-9 (197-317 cm). Das Interstadial beginnt mit einem Juniperus-Gipfel (21,8\%, auch Samen) und NBP-Anteilen von mehr als $70 \%$. Diese Juniperus Strauchphase (DA 6, 313-317 cm) leitete die Wiederbewaldung ein. Zunächst (DA 7, 301-313 cm) breiteten sich Baumbirken aus (bis $82 \%$ Pollen; Früchte und Fruchtschuppen in einzeln geborgenen Torfstücken), dann kamen Pinus (DA 8, 287-301 $\mathrm{cm}$, bis $25 \%$ ) und Picea hinzu (DA 9, 197-287 cm, bis $14 \%$, auch Nadeln). Larix hat vom DA 8 an eine geschlossene Kurve (bis 0,6\%). Die Lärche war, da sie im Pollenniederschlag immer stark unterrepräsentiert ist, offenbar im Gebiet anwesend. Artemisia-Pollen ist während der Birkenzeit (DA 7) besonders reichlich nachgewiesen (bis 5,6\%), wird aber in der Fichtenzeit seltener, vermutlich weil für diese Zeit das Temperaturoptimum und damit auch eine hohe Lage der Waldgrenze, oberhalb derer Artemisia wahrscheinlich wuchs, anzunehmen ist. Pollen von Bruckenthalia und Picea omoricoides konnte trotz sorgfältiger Suche nicht nachgewiesen werden.

Mit dem Wechsel von Niedermoortorf zu Seeschluff im DA 9 lassen sich auch wieder anspruchsvollere Gattungen wie Abies, Quercus, Ulmus u. a. nachweisen. Wieweit hier Umlagerung von Pollen (und anderen Pflanzenresten) vorliegt, ist nicht zu entscheiden. Es bleibt auch unklar, warum die Sedimente der DA 8-11 fast keine Samen, Früchte oder Hölzer enthalten.

DA 10 (152-197 cm). Gegen Ende des DA9 sinken alle Baumpollenkurven ab. Die Larix-Kurve setzt aus. Von den Gehölzarten ist im DA 10 nur noch Betula von einiger Bedeutung; ihre Kurve liegt mit Werten um $20 \%$ noch immer über denen von Pinus und Picea, aber die NBP-Werte erreichen jetzt stets mehr als $65 \%$. Waldlosigkeit, vielleicht sogar Baumlosigkeit ist angezeigt. Sogar die Artemisia-Werte gehen auf weniger als $1 \%$ zurück. Ephedra-Pollen tritt wieder auf. Erstmals gibt es hier (und im DA 11) eine Empetrum-Kurve (bis 2,6\%) und relativ hohe Calluna-Werte (bis 3,9\%).

DA $11(85-152 \mathrm{~cm})$. DA 11 umfaßt den jüngsten Abschnitt des Bohrprofils $\mathrm{H} 77$ sowie vier Proben aus noch jüngeren Horizonten des Aufschlußprofils $\mathrm{H} 75$. Pollenfloristisch unterscheidet er sich vom DA 10 nur wenig; er enthält aber den durch Humussubstanzen auffallend braun gefärbten, jedoch nicht torfigen 


\section{ASCHENHÜTTE/ Herzberg am Harz, 240 m NN}

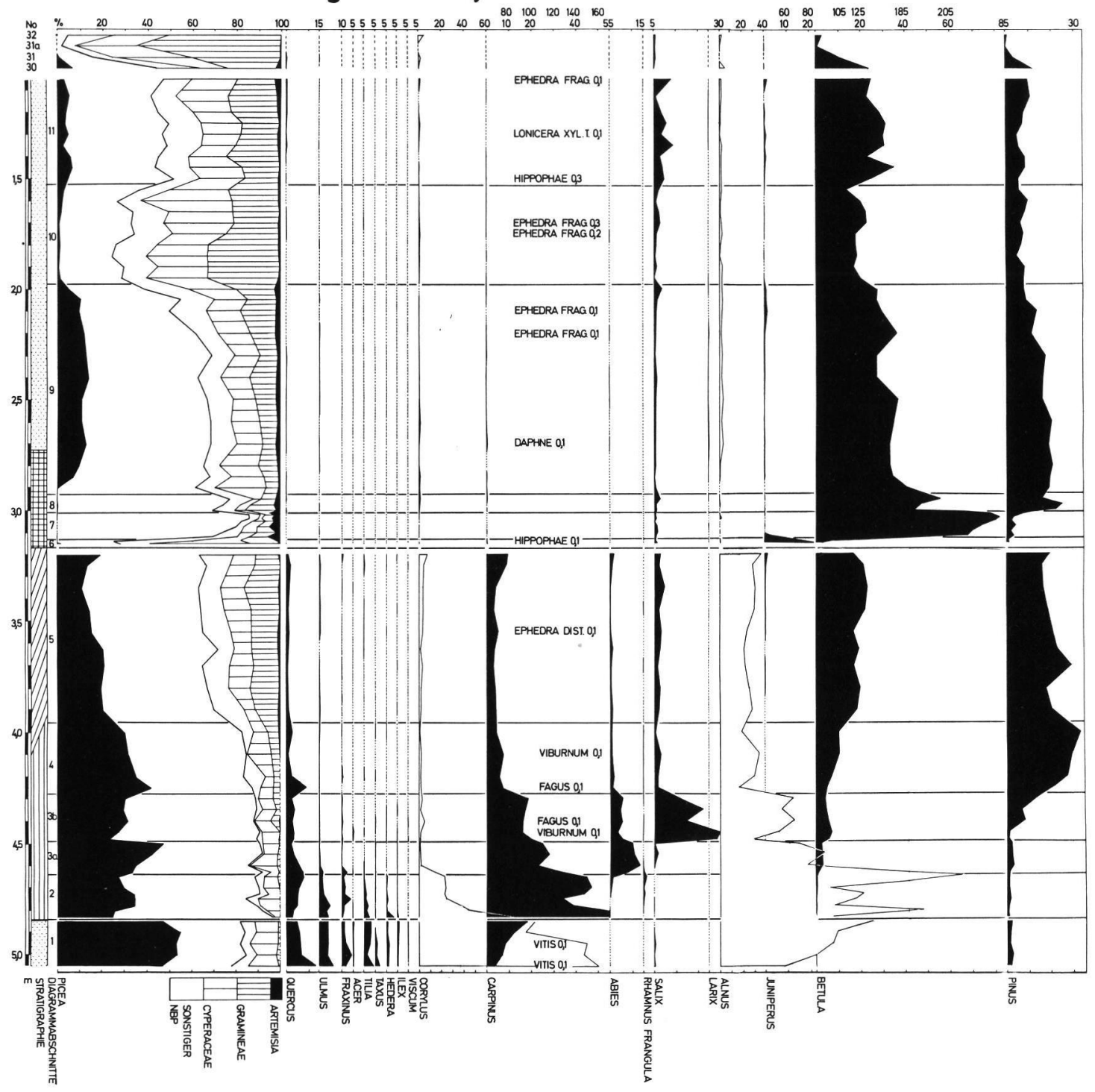

Abb. 4a: Pollendiagramm: Baum- und wichtige Nichtbaumpollenkurven.

Grundsumme: Landpflanzen ohne Alnus, Corylus und Farne. Stratigraphische Symbole wie in Abb. 2.

Abb. 4b: Großrest- und Nichtbaumpollendiagramm. Abkürzungen: Fr Frucht, FrS Fruchtschuppe, Stk Steinkern, Sa Samen, Ho Holz, Bl Blättchen, Nd Nadel, Kn Knospenschuppe, Skl Sklerotium. Stratigraphische Symbole wie in Abb. 2.

Fig. 4a: Pollen diagram: AP and main NAP curves.

Basic sum: terrestrial plants without Alnus, Corylus and ferns. Stratigraphical symbols as in Fig. 2.

Fig. 4b: NAP curves and distribution of seeds, fruits etc.

Abbreviations: Fr fruit, FrS fruit scale, Stk stony endocarp, Sa seed, Bl leaf, Nd needle, Kn bud scale, Skl Sclerotium.

Stratigraphical symbols as in Fig. 2. 


\section{ASCHENHÜTTE/ Herzberg am Harz, 240 m NN}

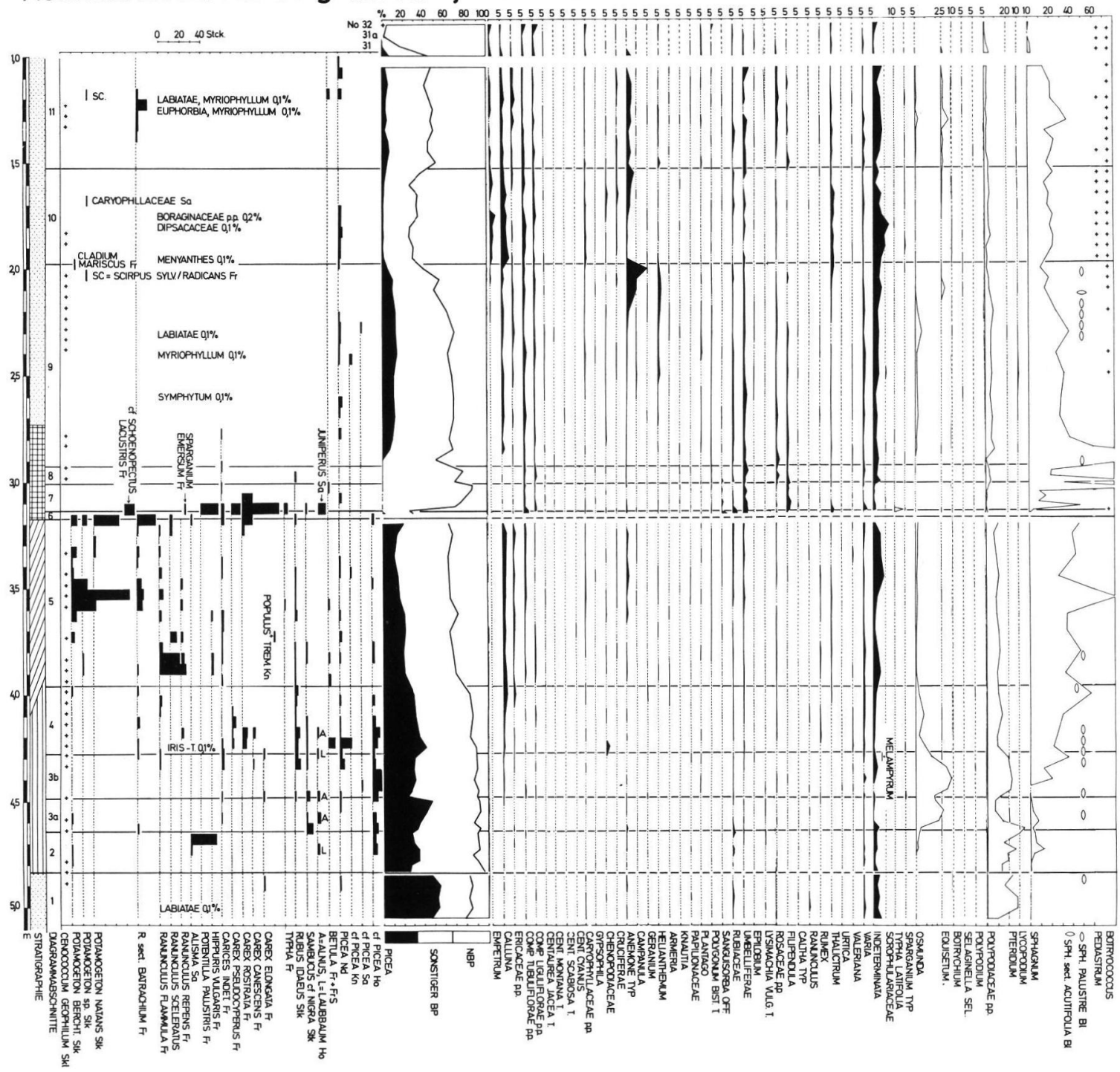

„Seggenhorizont”, einen Verlandungshorizont. Die an mehreren Bohrpunkten daraus entnommenen Pollenproben zeichnen sich durch nahe an $100 \%$ heranreichende NBP-Werte aus, zu denen Gramineae und Cyperaceae mehr als $90 \%$ beitragen.

Die älteren Proben des DA 11 fallen durch erhöhte Betula-, Salix- und Artemisia-Anteile auf. Darin mag eine Begünstigung dieser Arten zum Ausdruck kommen. Eine ähnliche Deutung der ebenfalls höheren Picea- und Quercus-Werte ist kaum möglich. Doch könnte Pollenumlagerung im Spiele sein. Sie setzt voraus, daß zur Zeit des DA 11 erodierbares Sediment mit den betreffenden Pollentypen vorhanden war. Im Falle von Quercus kämen dafür nur die Sedimente des DA 5 mit ihrem hohen Anteil schon einmal umgelagerten Pollens oder interglaziale Schichten in Frage. Letztere enthalten aber außer Quercus-Pollen noch andere Pollentypen, die im DA 11 nicht gefunden wurden. Deshalb kann eigentlich nur Sediment aus dem ersten Stadial (DA 5) erodiert worden sein, das dann auch zu den erhöhten Anteilen von Betula, Salix, Artemisia u. a. im DA 11 beigetragen haben dürfte. 


\section{Datierung der limnisch-telmatischen Sedimente}

\subsection{Eemzeitliche Sedimente}

Da der Aschenhütter Erdfall in die Oberterrassenkiese eingesenkt ist, die allgemein als elsterzeitlich eingestuft werden (DUPHORN 1974), können die interglazialen Sedimente nur jünger oder gleichalt wie das Holstein-Interglazial sein. Vegetationsgeschichtliche Kriterien (vor allem die zeitweilige CarpinusDominanz und das Fehlen sog. tertiärer Pollentypen einschließlich Eucommia und Pterocarya) führen zu der gleichen Folgerung und erlauben darüber hinaus, ein holsteinzeitliches Alter der Sedimente auszuschließen (vergl. dazu CEPEK; ERD \& ZWIRNER 1981; ERD 1970; GrÜGER 1968; MeNKE 1968; MÜller 1974; Stremme \& MENKE 1980; TURNER 1975 u. a.). Damit entfällt auch der Vergleich mit der cromerzeitlichen Vegetationsabfolge, die vom nur $12 \mathrm{~km}$ entfernten Bilshausen (MÜLLER 1965) bekannt ist und die sich trotz zeitweiliger Hainbuchendominanz in wichtigen Merkmalen von der bei Aschenhütte nachgewiesenen unterscheidet (gleichzeitige Ausbreitung von Carpinus und Abies, völlige Bedeutungslosigkeit von Corylus usw.).

Somit muß die ältere Waldzeit von Aschenhütte in den Endabschnitt der Eem-Warmzeit gestellt werden. Die hier erkennbare Vegetationsentwicklung stimmt mit der von den nächstgelegenen eemzeitlichen Untersuchungspunkten (Wallensen: RABIEN 1953; Lüneburger Heide: MÜLLER 1974; BEHRE 1962, 1974; BEHRE \& LADE 1986) in folgenden Merkmalen überein: Der Hainbuchenzeit geht eine Zeit mit Haselanteilen von meistens mehr als $100 \%$ voraus. Die Hainbuche erreicht überall Werte von mehr als $40 \%$. Spätestens in der frühen Hainbuchenzeit werden Quercus, Ulmus und Tilia bedeutungslos. Die TaxusKurve erreicht ihr Maximum (Wallensen 23,2\%: GRÜGER 1968) vor Beginn der Hainbuchenzeit. Während dieser war Taxus ohne jede Bedeutung. Höhere Abies-Werte treten erst in der ausklingenden Hainbuchenzeit auf. Die höchsten Tannenwerte findet man im Bereich der Mittelgebirge (Wallensen, Aschenhütte).

Beim Vergleich der oben zitierten Diagramme ergeben sich größere Unterschiede nur bei Arten, die standörtlich bedingt - lokal größere Bestände zu bilden vermögen wie Alnus und Salix und bei Picea.

Picea-Pollen ist in allen Vergleichsdiagrammen schon in den Abschnitten der EMW-Zeit verzeichnet. Ihre Höchstwerte erreicht die Fichte aber erst in der mittleren Hainbuchenzeit oder danach. So liegen die Fichtenwerte in Wallensen während der Hainbuchenzeit unter $10 \%$ und ihr Maximum (fast $30 \%$ ) am Ende derselben. Im Pollendiagramm Aschenhütte dagegen bewegt sich die Fichtenkurve schon während der Hainbuchenzeit um die $30 \%$-Linie und ihr Höchstwert $(55,2 \%)$ fällt in den DA 1 (beginnende Hainbuchenausbreitung). Wie Funde von Nadeln, Samen und Knospenschuppen bei Wallensen (160 m NN, RABIEN 1953) und in der Lüneburger Heide (50 bis $60 \mathrm{~m} \mathrm{NN}$, BEHRE 1962) beweisen, war die Fichte trotz niedriger Pollenwerte - schon während der EMW-Zeit in Norddeutschland anwesend. Wo sie schon vor der Hainbuchenausbreitung hohe Werte erreicht wie im Profil Westerland-Friedrichshain auf Sylt, werden diese auf lokale Bestände der Fichte an Moorrändern oder in Bruchwäldern zurückgeführt (AVERDIECK 1967; BEHRE 1962; HALLIK 1953, u. a.). Bei Aschenhütte kam die Fichte mindestens seit Beginn der Hainbuchenzeit in tiefen Lagen vor, wie Nadeln beweisen. Ein Teil des nachgewiesenen Pollens wird aus diesen Fichtenbeständen stammen, ein anderer aber aus den nahen Bergwäldern des Harzes.

Pollendiagramme süddeutscher Vorkommen, die der letzten Warmzeit zugeschrieben werden, zeigen, daß hier der Hainbuchenausbreitung eine Zeit hoher Fichtenwerte vorausging (z. B. Leutenbach bei Stuttgart: FREISING \& FILZER 1978, Zeifen bei Laufen a. d. Salzach: JUNG, BEUG \& DEHM 1972). Nicht frühzeitig hohe, sondern die trotz der Anwesenheit der Fichte in Norddeutschland so lange niedrig bleibenden Fichtenwerte wären zu diskutieren!

\subsection{Weichselzeitliche Sedimente}

Da die Sedimente im Liegenden eemzeitlichen Alters sind, müssen die hangenden Schichten (ab DA 5) aus der Weichsel-Kaltzeit stammen. Diese Deutung wird durch die Verzahnung der hangenden Schichten mit der für das niedersächsische Bergland typischen Weichsel-Lößabfolge bestätigt (ROHDENBURG \& MEYER 1966; RICKEN 1983; RICKEN \& MEYER 1982). Die im kaltzeitlichen Abschnitt nachgewiesene Waldzeit (DA 6-9) muß einem der beiden frühweichselzeitlichen Interstadiale entsprechen; denn am Ende des Frühweichsels verschwand die Fichte aus NW-Europa und erschien hier erst im späten Holozän wieder.

MenKe und TynNi (1984) u. a. Autoren unterscheiden in Norddeutschland zwei Frühweichsel-Interstadiale, das (ältere) Brörup-Interstadial und das (jüngere) Odderade-Interstadial. Ein schwacher Klimarückschlag innerhalb des Brörup-Interstadials läßt dieses in manchen Pollendiagrammen zweigeteilt erscheinen. Wenn diese beiden Teile des Brörup-Interstadials als eigenständige Interstadiale aufgefaßt werden, bezeichnet man das ältere als Amersfoort- und das 
jüngere als Brörup-Interstadial (sensu ZAGWIJN 1961). Letzteres stellt also nur einen Teil des Brörup-Interstadials sensu ANDERSEN (1961) an der Typus-Lokalität dar. Wie diese Interstadiale miteinander zu korrelieren und $\mathrm{zu}$ benennen seien, ist eingehend diskutiert worden (ANDERSEN 1961; AVERDIECK 1967; BeHRE \& LADE 1986; ERD 1973; MENKE 1976; MENKE \& TYNNI 1984; SCHNEEKLOTH 1966).

$\mathrm{Zu}$ entscheiden, welchem der beiden FrühweichselInterstadiale das Interstadial von Aschenhütte zuzuordnen ist, fällt schwer; denn die Vegetationsentwicklung ist im Brörup-Interstadial offenbar ganz ähnlich verlaufen wie im Odderade-Interstadial. Zwar hat es den Anschein, als habe die brörupzeitliche Flora mehr thermisch anspruchsvolle Arten enthalten als die des Odderade-Interstadials, aber dieses Merkmal mag bei Aschenhütte wegen seiner vergleichsweise hohen Lage (240 m NN) am Harzrand (Waldgrenze!) und wegen des hier relativ kontinentalen Klimas nicht mehr erkennbar sein. (Die Vergleichsdiagramme stammen von Kittlitz in der Niederlausitz abgesehen - sämtlich von küstennahen, tiefgelegenen Untersuchungspunkten). Ein Klimarückschlag, der das Aschenhütter Interstadial teilt und als Hinweis auf ein brörupzeitliches Alter desselben dienen könnte, ist kaum zu erkennen. Er müßte sich im Pollendiagramm vor dem Anstieg der Picea-Kurve und zwar im DA 8 bemerkbar machen. Deutet man aber den Juniperus-Gipfel des DA 6 als Folge dieser brörupzeitlichen Klimaverschlechterung, dann muß man folgern, daß im Profil Aschenhütte die Sedimente des Amersfoort-Interstadials fehlen. Ordnet man das Interstadial dem Odderade zu, dann fehlen die Sedimente des Brörup-Interstadials (sensu Andersen) und wenigsten eines der zugehörigen Stadiale. Da das Becken des Aschenhütter Sees zu dieser Zeit noch mehr als $2 \mathrm{~m}$ tief war (vergl. Kap. 8) und weil es kaum vorstellbar ist, daß im Verlaufe von mindestens 15000 Jahren, die das BrörupInterstadial und das darauf folgende Rederstall-Stadial bis zum Beginn des Odderade-Interstadials gedauert haben, nichts in das Erdfallbecken sedimentiert worden wäre, nehmen wir an, daß die Waldzeit der DA 7-9 das Brörup-Interstadial sensu ANDERSEN repräsentiert und daß die im $\mathrm{DA} 8$ vorübergehend höheren NBP- und Betula-Anteile einziger Ausdruck des brörupzeitlichen Klimarückschlags sind.

Die Schichtlücke zu Beginn des Interstadials ist auf ein Absinken des Wasserspiegels im Herning-Stadial zurückzuführen (vergl. Kap. 8). Sie repräsentiert einen Zeitabschnitt unbekannter, aber vermutlich relativ kurzer Dauer. Dagegen umfaßt die auf den DA 11 folgende Schichtlücke einen langen Zeitabschnitt; denn noch vor Beginn des nächsten Interstadials (Odderade) war der Aschenhütter See verlan$\operatorname{det}(\mathrm{Abb}$. 2: $\mathrm{S}=$ Seggen-Verlandungshorizont), und die hangenden Fließerden und Lößlehme sind mittelund hochglazialen Alters.

\section{Kohlenstoff- und Stickstoff-Gehalte}

Der relative Gehalt an organisch gebundenem Kohlenstoff und Stickstoff in Böden und Torfen, das $\mathrm{C} / \mathrm{N}$-Verhältnis, ist im wesentlichen durch den Zersetzungsgrad der organischen Substanz bestimmt (SCHACHTSCHABEL et al. 1976; NAUCKE 1980) und weniger auf differierende primäre Gehalte an Kohlenstoff und Stickstoff zurückzuführen. Wird beim biologischen Abbau der organischen Substanzen zeitweise mehr Kohlendioxid freigesetzt als Stickstoff, dann wird das $\mathrm{C} / \mathrm{N}$-Verhältnis enger. Deshalb haben die Mudden und Torfe von Aschenhütte ein $\mathrm{C} / \mathrm{N}$ Verhältnis zwischen 15 und 65, wenig veränderte Hölzer aus dem Eem-Interglazial und dem FrühweichselInterstadial aber ein solches zwischen 100 und 200.
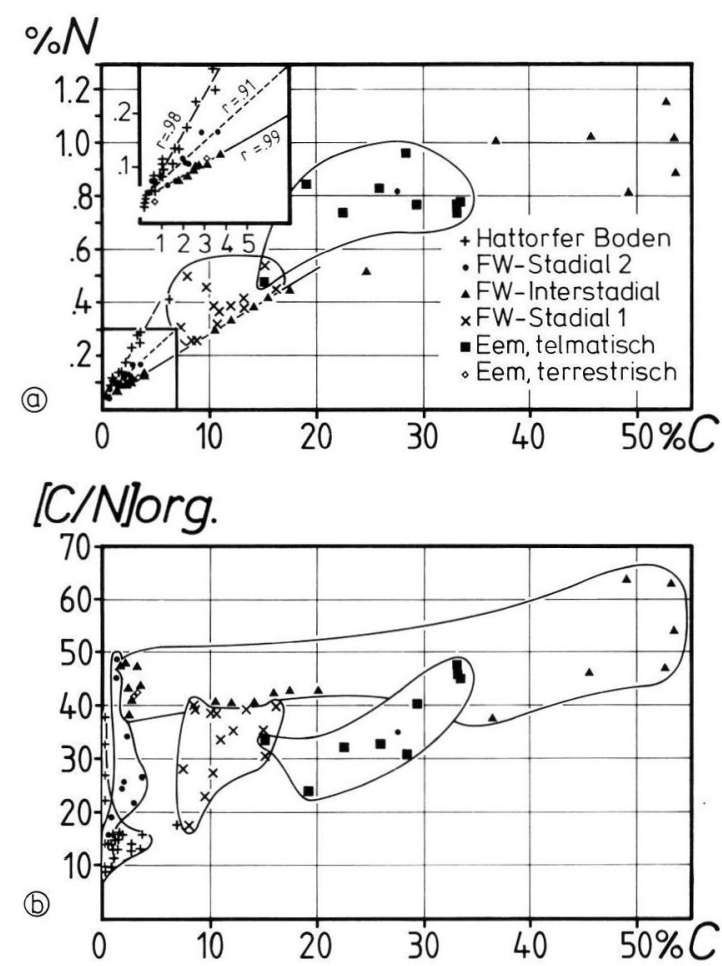

Abb. 5: a) Gesamtstickstoffgehalt in Abhängigkeit vom Kohlenstoffgehalt der karbonatfreien Proben (Gew. \%).

b) Kohlenstoff/Stickstoffverhältnis der organischen

Substanz in Abhängigkeit vom Kohlenstoffgehalt der karbonatfreien Sedimente.

Fig. 5: a) Total nitrogen-versus-carbon content of the carbonate-free sediments (weight \%).

b) Carbon / nitrogen ratio of the organic matter versus carbon content of the carbonate-free sediment. 
Bei Laub- und Nadelstreu liegt es gewöhnlich zwischen 40 bis 60 , bei Gräsern zwischen 50 und 100 (SCHACHTSCHABEL et al. 1976). Da in die aus Bulkanalysen gewonnenen $\mathrm{C} / \mathrm{N}$-Verhältnisse die Anteile des anorganisch gebundenen Stickstoffs eingehen, muß eine Korrektur durchgeführt werden, um das $\mathrm{C} / \mathrm{N}$ Verhältnis der reinen organischen Substanz zu erhalten. Für diesen Zweck wurden für die verschiedenen, durchweg karbonatfreien Sedimenttypen die Gesamtgehalte von Kohlenstoff gegen Stickstoff aufgetragen (Abb. 5a). Dabei lassen sich mit Ausnahme der umgelagerten Schluffmudden des 1. Stadials (HerningStadial) Sedimente mit einem Gesamtkohlenstoffgehalt unter $20 \%$ linear korrelieren (Korrelationskoeffizienten: 0,91 bis 0,99). Bei kohlenstofffreien Sedimenten ergibt sich aus den Korrelationen die mineralische Stickstoffbindung für die limnisch-telmatischen Sedimente des Aschenhütter Sees mit durchschnittlich $0,044 \mathrm{Gew}$. \%. Dagegen beträgt der mineralische Stickstoffanteil der Lößböden des Stillfried-B-Interstadials (Hattorfer Boden) nur etwa die Hälfte des anorganischen Stickstoffanteils der limnisch-telmatischen Sedimente (Tab. 1), was vermutlich mit einem höheren Gehalt derselben an adsorptiven Tonmineralen zu erklären ist. Unter Subtraktion des mineralischen Stickstoffanteils wurde aus den Gesamtbestimmungen das $\mathrm{C} / \mathrm{N}$-Verhältnis der rein organischen Substanz berechnet. Bei niedrigen Gesamtkohlenstoffgehalten macht sich die Korrektur in einer deutlichen Erhöhung des $\mathrm{C} / \mathrm{N}$-Verhältnisses bemerkbar (Abb. 3). Im folgenden werden drei Aspekte näher erläutert.
1. Wie Abb. 5b zeigt, besitzen die verschiedenen Sedimenttypen von Aschenhütte sowohl charakteristische organische $\mathrm{C} / \mathrm{N}$-Verhältnisse als auch charakteristische Gesamtkohlenstoffgehalte (Tab. 1). Man erkennt eine Tendenz zu weiten $\mathrm{C} / \mathrm{N}$-Verhältnissen, d. h. schlechterer Mineralisation der organischen Substanz, mit steigendem Kohlenstoffgehalt der Sedimente. Dieser Trend ist auch in rezenten Böden festgestellt worden (SCHACHTSCHABEL et al. 1976) und ist darauf zurückzuführen, daß bei behinderter Zersetzung mehr organisches Material akkumuliert wird. Umgekehrt ist es möglich, daß bei großer Bioproduktion nur ein Teil der anfallenden Biomasse abgebaut werden kann.

2. Zwischen Sedimenttyp und C/N-Verhältnis besteht eine deutliche Abhängigkeit. Torfe des Interglazials und des Interstadials zeigen aufgrund konservierender Bildungsbedingungen eine nur geringe Zersetzung. Seesedimente der Stadiale 1 und 2 haben mittelgroße Zersetzungsbeträge, da die Mineralisation am Seeboden in belüftetem Wasser stattfinden konnte. Der See war mit einer Wassertiefe von wenigen Metern (vergl. Kap. 8) offenbar zu flach, um eine dauerhafte Temperaturschichtung im Wasserkörper auszubilden. Die Mineralisationsbeträge deuten auf dimiktische bis polymiktische Verhältnisse. Vergleichsweise sehr hohe Beträge der Mineralisation werden im Hattorfer Boden erreicht. Obwohl dieser Löß-Paläoboden als Stillfried-B-Äquivalent aufgrund seiner Kryoklastik und Solifluktionserscheinungen einem Tundrenklima zugeordnet werden muß, liegt sein $\mathrm{C} / \mathrm{N}$-Verhältnis,

Tab. 1: Kohlenstoff- und Stickstoffgehalte der karbonatfreien Sedimente des Aschenhütter Sees und in den Löß-Paläoböden

Tab. 1: Carbon and nitrogen contents of carbonate-free samples recovered from sediments of the former Lake Aschenhütte and from loess paleosoils

mineralischer

Stickstoffanteil

(Gew. \%)
Kohlenstoffgehalt

(Gew. \%)
C/N-Verhältnis der organischen Substanz

\begin{tabular}{lcccc}
\hline FW-Interstadial & & & \multicolumn{2}{c}{ Mittelwert } \\
Torfe & & & $46-64$ & 55 \\
$\quad$ Mudden & 0,043 & $45-54$ & $38-48$ & 43 \\
Eem-Interglazial & & $2-36$ & $24-48$ & 37 \\
1. FW-Stadial & & $15-34$ & $17-40$ & 33 \\
2. FW-Stadial ${ }^{*}$ ) & 0,045 & $1-4$ & $15-49$ & 30 \\
Hattorfer Boden $^{+}$) & 0,024 & $0-7$ & $9-38$ & 15 \\
\hline
\end{tabular}

*) ohne Seggen-Verlandungshorizont

+) aus Lößbohrungen im südlichen Harzvorland 
verglichen mit mitteleuropäischen Böden, zwischen den Humusformen Mull $(\mathrm{C} / \mathrm{N}=10)$ und Moder $(\mathrm{C} / \mathrm{N}=20)$.

3. Die Frage, ob der Grad der Zersetzung von Klimavariationen abhängig ist, muß offen bleiben. Im Aschenhütter See scheinen Sedimente mit gleichem Kohlenstoffgehalt höhere Zersetzungsbeträge aufzuweisen, wenn sie unter ungünstigeren klimatischen Bedingungen abgelagert wurden. Dies könnte mit niedrigem Wasser und dem damit verbundenen besseren Sauerstoffangebot zusammenhängen (vergl. Kap. 8).

\section{Paläoböden}

Von überregionaler Bedeutung ist die Verzahnung und Überlagerung der pollenanalytisch datierten Sedimente mit Löß-Paläoböden (vergl. Abb. 2 und 6):
1. Am nordwestlichen Seeufer werden $\operatorname{der} A_{1^{-}}$und $B_{t}$-Horizont einer Parabraunerde von auf den Erdfall transgredierenden Mudden überlagert, welche nach dem pollenanalytischen Befund in Bohrung 82/8 im ausgehenden Eem-Interglazial (DA 4/5) und in Bohrung $82 / 1$ im beginnenden Stadial 1 (DA 5) gebildet worden sind (Abb. 6). Folglich handelt es sich hierbei um den Eemboden. Seine Vergleyungserscheinungen sind mäßig; der $\mathrm{A}_{\mathrm{\Gamma}}$-Horizont enthält - wie auch andere Eemböden am südlichen Harzrand (RICKEN 1983) - nur geringe Anteile an Holzkohlen und $\mathrm{MnO}_{2}$-Konkretionen. Damit kann als sicher gelten, daß der zugehörige Bleichhorizont noch in der Endphase des Eem-Interglazials entstanden ist. Die Bleichung des Oberbodenhorizontes wurde vermutlich durch die in dieser Zeit erfolgende Änderung der Vegetation zu ericaceen-reichen Nadelwäldern begünstigt (vergl. Abb. 4).

2. Pedologische Äquivalente der ersten Stadialphase konnten nicht erkannt werden. Jedoch gehen Torfe

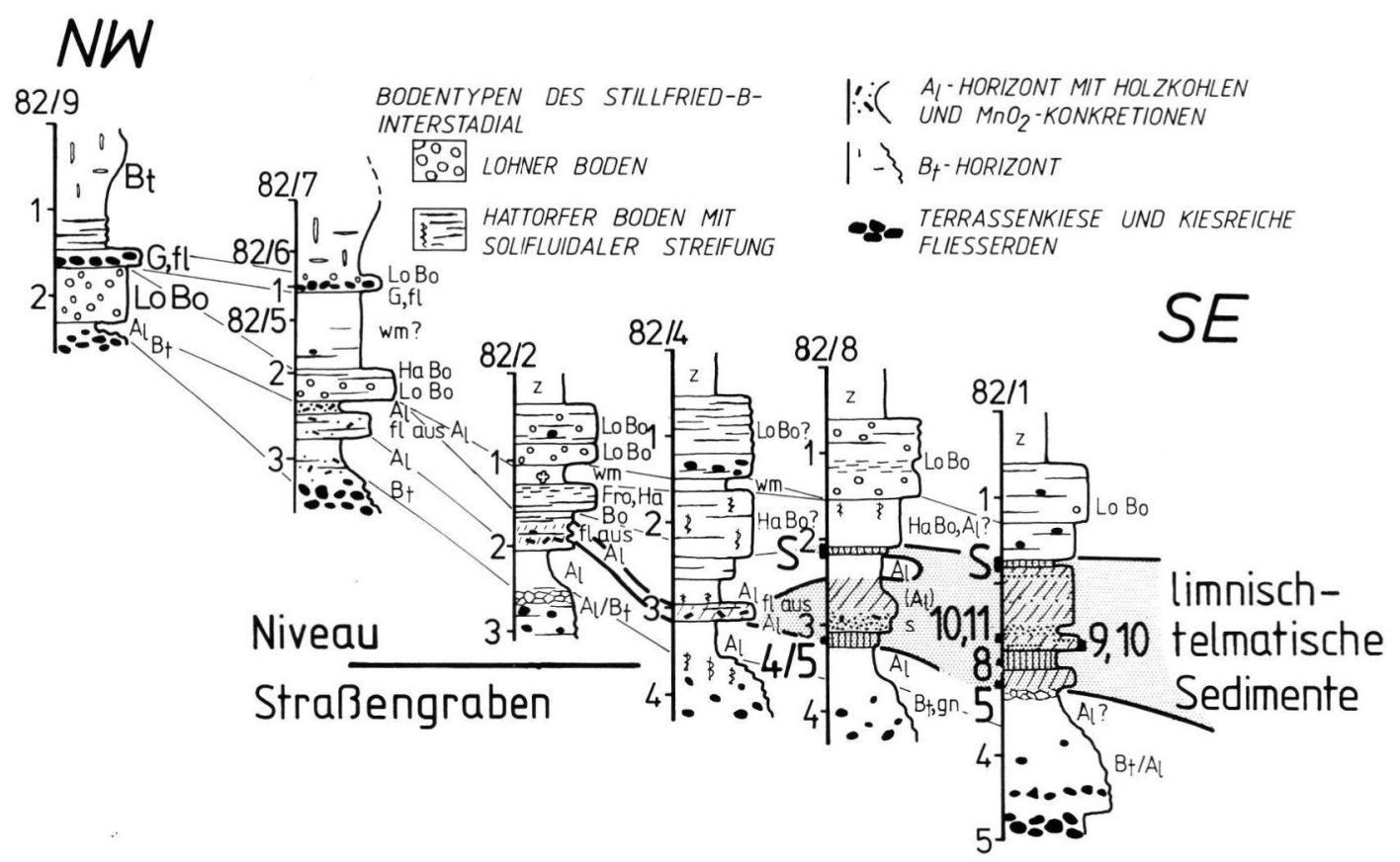

Abb. 6: Verzahnung der limnischen und telmatischen Sedimente des ehemaligen Aschenhütter Sees mit Lößpaläoböden des Eem-Interglazials und des Frühweichsels am nordwestlichen Seeufer.

Bezeichnung der Diagrammabschnitte wie in Abb. 2. Abkürzungen: G Kies, s Sand, fl Fließerde,

Fro Frostplattung, wm fragliches Lößmaterial des Mittelweichsels, z gestörte Lagerung,

$B_{t}$ Tonanreicherungshorizont, $A_{l}$ Tonverarmungshorizont, Habo Hattorfer Boden, Lobo Lohner Boden.

Fig. 6: Transition between limnic and telmatic sediments and paleosoils of the Eemian interglacial and the Lower Weichselian at the northwestern shore of the former Lake Aschenhütte. Numbers of cores and pollen zones are the same as in Fig. 2. Abbreviations: $G$ gravel, $s$ sand, fl solifluction horizon, Fro frost cleavage, wm loess of the Middle Weichselian period, uncertain, $\mathrm{z}$ disturbed stratification, $\mathrm{B}_{\mathrm{t}}$ horizon of clay mineral enrichment, $\mathrm{A}_{\mathrm{l}}$ horizon of clay mineral depletion, Habo Hattorf soil, Lobo Lohne soil (both soils are equivalent to the Austrian Stillfried-B-horizon). 
und Mudden des Frühweichsel-Interstadials (DA 6-9) in den Bohrungen $82 / 1,2,4$ und 8 in eine nur $20 \mathrm{~cm}$ mächtige, humose Oberboden-Fließerde über (Abb. 6). Erst während des 2. Stadials wird dagegen verstärkt Bleichhorizontmaterial in den Aschenhütter See verlagert und das Ufer gegen den See vorgebaut (Abb. 6). Dabei muß offen bleiben, welchen Anteil hierbei Verspülung und welchen Solifluktion hatten.

3. Die limnischen Sedimente des Aschenhütter Sees werden von zwei Lößboden-Fließerdelagen des Stillfried-B-Interstadials überlagert, welche jeweils basale Sohlen aus Kiesmaterial der Oberterrasse aufweisen. Darüber folgen noch $2 \mathrm{~m}$ entkalkter Jungweichsellöß, der von der holozänen (pedogenen) Tondurchschlämmung kräftig überprägt ist. Die kryoklastischen Solifluktionsböden des Stillfried-B-Interstadials stellen Mischformen der Naßfazies (Hattorfer Boden; RICKEN 1983) und der Trockenfazies dar (Lohner Boden; SCHÖNHALS et al. 1964; ROHDENBURG et al. 1966). Dabei überwiegt hier anteilmäßig der Hattorfer Boden, welcher durch Marmorierung und konzentrische Eisen-und Manganoxidfällungen um fossile Wurzelröhren gekennzeichnet ist. Der Lohner Boden weist dagegen eine braune Färbung und $\mathrm{FeOOH}$ Konkretionen auf.

Wenn auch in reduzierter Mächtigkeit, so sind im Aschenhütter Erdfall alle wichtigen Paläoböden und Lößhorizonte des Jungpleistozäns vorhanden, nämlich: Eemboden, Bleichhorizonte der Frühweichselzeit, Mittelweichselböden (hier mit den Bodentypen des Stillfried-B-Interstadials) und Jungweichsellöß. Die pollenanalytische Datierung der sich mit den Lößderivaten verzahnenden limnisch-telmatischen Sedimente bestätigt die grundsätzliche Richtigkeit der von der Lößforschung aus anderen Überlegungen getroffenen zeitlichen Einstufung der Paläoböden (SCHÖNHALS et al. 1964; ROHDENBURG et al. 1966; HAASE et al. 1970; RICKEN \& MEYER 1982; RICKEN 1983).

\section{Limnische Entwicklung und Spiegelschwankungen}

Die Verbreitung der Sedimenttypen, die Schichtlücken und einige Besonderheiten im Pollendiagramm sind zum Teil auf Spiegelschwankungen des Aschenhütter Sees zurückzuführen. Die Spiegelschwankungen lassen sich aus der Sedimentverteilung und aus dem Verlauf der Isochronen aus Abb. 2 gewinnen.

Die limnisch-telmatische Sedimentation im Erdfall begann mit der Bildung von schluffigem Niedermoortorf am Ende der Eichenmischwaldphase des EemInterglazials (DA 1). Davor hat eine Phase unbekann- ter Dauer gelegen, während der der vermutlich zunächst noch schlotartige Erdfall zum großen Teil mit Kiesbrekzien aus Oberterrassenmaterial verfüllt worden ist. Der Erdfall entstand wahrscheinlich in dem Intervall zwischen der späten Saalezeit und der Zeit des DA 1.

Während des jüngeren Eems lag der Wasserspiegel tief (die Großrestanalyse erbrachte für die DA $1-3$ nur wenige Nachweise von Sumpf- und Wasserpflanzen); doch stieg er bis zum Ende des Eem-Interglazials (DA 4/5) beträchtlich an (Nachweise von Arten nasser Standorte, vor allem Seggen, werden häufiger). Dabei transgredierten die eemzeitlichen Sedimente gegen die Erdfallränder und überlagerten dort den im späten Eem-Interglazial gebildeten Bleichhorizont (vergl. die in Abb. 2 in Kreisen eingetragenen Nummern der DA der jeweils ältesten eemzeitlichen Sedimente).

Im ersten Frühweichsel-Stadial (DA 5) wurde ein relativer Wasserhöchststand erreicht $(+3 \mathrm{~m}$, zahlreiche Reste von Wasserpflanzen). Dabei wurde ein Teil der Sedimente des Eem-Interglazials von den Erdfallrändern in das Seezentrum umgelagert und zusammen mit stadialen Schluffen erneut sedimentiert (vergl. die noch hohen, kontinuierlich abnehmenden Kohlenstoffgehalte der stadialen Sedimente, das ähnliche $\mathrm{C} / \mathrm{N}-$ Verhältnis von stadialen und eemzeitlichen Sedimenten und den relativ hohen Anteil thermisch anspruchsvoller Baumarten neben den neu auftretenden kaltzeitlichen Pflanzenarten).

Vor dem Beginn des Brörup-Interstadials war der Aschenhütter See im Bereich der Profillinie (vergl. Abb. 2) offenbar trocken, so daß zunächst keine interstadialen Sedimente abgelagert wurden: selbst in den tiefsten Bereichen des Aschenhütter Sees (Bohrung $\mathrm{H} 77$ ) fehlt der Beginn der das Interstadial einleitenden Juniperus-Phase (Abb. 4).

Während des Brörup-Interstadials (DA 6-9) stieg der Wasserspiegel erneut an. Im noch flachen Wasser kam es bei der Wiederbewaldung zunächst zur Bildung von Birkenbruchwaldtorf. Mit dem Anstieg des Wasserspiegels transgredierte die Torfbildung gegen die Erdfallränder (vergl. die Diagrammabschnittsnummern der Basishorizonte am südöstlichen Seeufer, Abb. 2). Der stetige Rückgang der Kohlenstoffgehalte in den Schluffen und Mudden des DA 9 (Bereich der Seemitte) kann als ein weiterer Hinweis auf den interstadialen Anstieg des Seespiegels gewertet werden (vergl. Abb. 7). Die Großrestanalyse lieferte - wahrscheinlich aufgrund spezifischer (schlechter) Erhaltungsbedingungen - nur wenig Hinweise auf die Art der lokalen Vegetation. Wegen ihrer Feinschichtung müssen die Sedimente des DA9 jedoch als limnisch Bildungen aufgefaßt werden. 


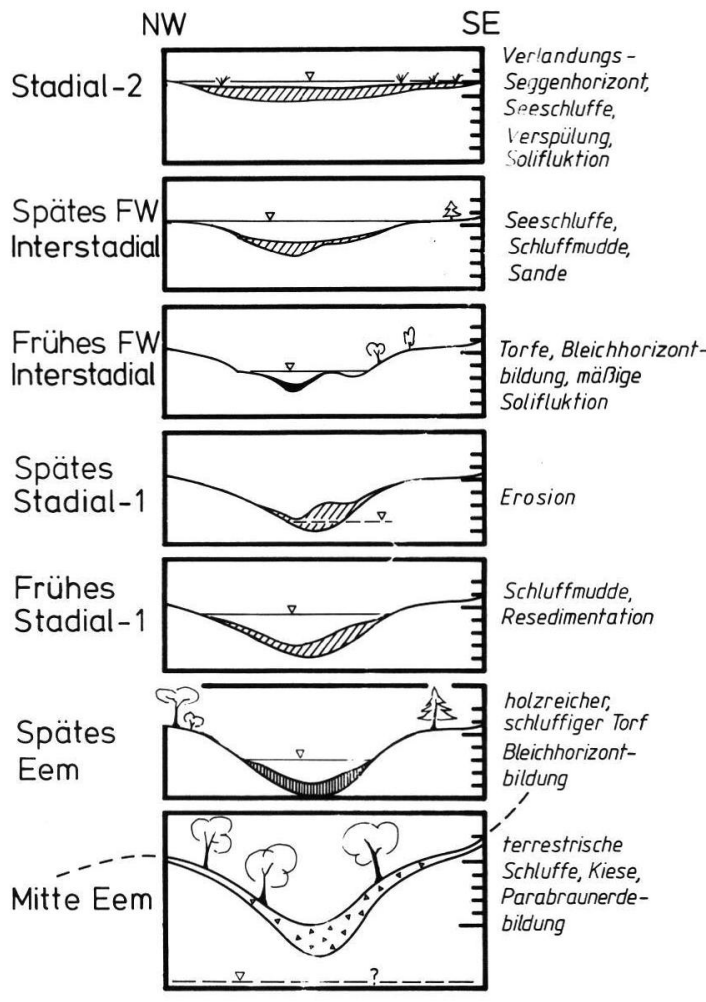

Abb. 7: Sedimentation und Spiegelschwankungen im ehemaligen Aschenhütter See.

Fig. 7: Sedimentation and changes of the water level in the former lake Aschenhütte.

Im nachfolgenden 2. Stadial (DA 10-11) stieg der Wasserspiegel noch über die Abrasionsfläche des Südwestufers an. Sehr wahrscheinlich blieb der Wasserstand in etwa konstant, da sich ein Überlauf an der Flachuferseite im SW bildete. Der Aschenhütter See verlandete unter Bildung eines an Huminstoffen reichen Horizontes, der sich pollenanalytisch durch sehr hohe Gramineen- und Cyperaceenwerte auszeichnet. Der Gehalt an organischer Substanz nimmt in diesem Horizont noch einmal kurzfristig zu. Der Pollenbefund zeigt Waldlosigkeit an (DA 11).

Aus der Sedimentverteilung und den dargestellten Spiegelschwankungen ergeben sich die für den Bereich der Seemitte dargestellten Kurven der Wasserspiegelhöhe und der kumulativen Sedimentmächtigkeit (Abb. 8). Für die Ermittlung der letzteren wurden die heutigen Mächtigkeiten von Detritusmudde und Torf mit den Faktoren 1,5 (Eem) und 2 (Brörup) multipliziert, um die erfahrene Setzung wieder auszugleichen. Dieser Setzungs- oder Kompaktionsbetrag wurde durch Messungen an 53 aus dem Torf stammenden Hölzern mit jetzt elliptischen Querschnitten bestimmt.

Es ergeben sich zwei relative Wasserhöchststände, nämlich für den Beginn des ersten FrühweichselStadials (DA 5, Herning-Stadial) und für das mittlere Brörup-Interstadial (DA 7-8). Dagegen war der untersuchte Bereich des Aschenhütter Sees am Beginn des Brörup-Interstadials trocken (DA 5/6). Da die Wasserstandskurve im Prinzip ähnlich wie die Kurve der kumulativen Sedimentmächtigkeit ansteigt, kann vermutet werden, daß durch die abdichtende Wirkung der limnischen Sedimente ein lokaler Wasserspiegel gebildet wurde, dessen Spiegelhöhe sich klimatisch bedingt von Zeit zu Zeit änderte. Die hohen Seespiegelstände zu Beginn der Weichsel-Kaltzeit und während der jüngeren Abschnitte des BrörupInterstadials repräsentieren demnach feuchte Perioden mit relativ geringen Verdunstungsraten. Da am Ende des Herning-Stadials trotz geringerer Verdunstung (kälteres Klima und Waldlosigkeit) eine Absenkung des Seespiegels erfolgte, muß dieser Zeitabschnitt ein relativ trockener gewesen sein. Eine ähnliche Deutung von Spiegelschwankungen in pleistozänen Seeablagerungen Norddeutschlands geben MANIA et al. (1970) und USINGER (1981).

Die tiefe Lage der Grundwasseroberfläche während des Eem-Interglazials (vor dem Beginn der limnischtelmatischen Sedimentation im Aschenhütter Erdfall) ist - in Analogie zu den heutigen Verhältnissen am südlichen Harzrand - eine Folge von interglazialer Subrosionstätigkeit und fluviatiler Erosion (Ricken

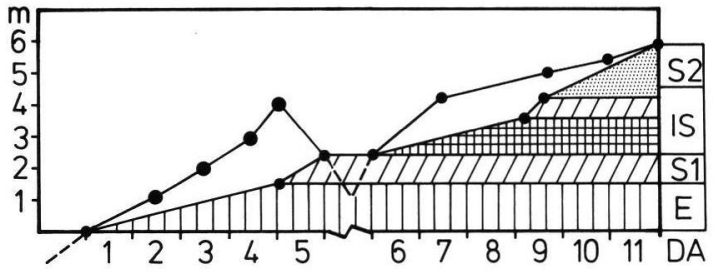

Abb. 8: Kumulative Sedimentmächtigkeit und Spiegelhöhen des ehemaligen Aschenhütter Sees für den Bereich der Seemitte (in Metern) in Abhängigkeit von der Zeit (dargestellt als Diagrammabschnitte $=\mathrm{DA}$, gemäß Fig. 4). Abkürzungen: E Eem-Interglazial, S1 erstes Stadial, S2 zweites Stadial, IS Frühweichsel-Interstadial

(Brörup). Signaturen der Sedimente wie in Abb. 3.

Fig. 8: Cumulative thickness of the sediment and water level changes of former Lake Aschenhütte versus time, using the pollen zones (DA) of Fig. 4a., based on data from the middle of the lake. Abbreviations: E Eemian interglacial; S1 first stadial, S2 second stadial and IS Lower Weichselian interstadial (Brörup) periods. The symbols used for the sediments are the same as in Fig. 3. 
1982). Beide Prozesse führten damals wie heute zu einer beträchtlichen Erniedrigung des Talauenniveaus und damit auch zur Erniedrigung der Grundwasseroberfläche. Wieweit die Bildung eines Sees im Aschenhütter Erdfall mit der möglichen Aufschüttung des ca. $150 \mathrm{~m}$ entfernten Kieskörpers der Niederterrasse zusammenhängt, muß offen bleiben. In diesem Falle ergäbe sich wegen der relativ hohen Lage des Aschenhütter Sees über dem eemzeitlichen Talauenniveau ein verhältnismäßig steiler, unrealistischer Anstieg der Grundwasseroberfläche. Aufgrund der Verzahnung mit Paläoböden wird vermutet, daß ein beträchtlicher Teil der Aufschüttung des Kieskörpers der Niederterrasse in den Solifluktionsphasen des Frühweichsels erfolgte (RICKEN 1982).

Zusammenfassend läßt sich feststellen: Die Fichte war während der Eemwarmzeit nicht nur in den Bergwäldern des Harzes vertreten, sondern kam - wenigstens an besonders feuchten Stellen - auch in den Tieflagen vor. Der mancherorts festgestellte brörup-zeitliche Klimarückschlag macht sich im Pollendiagramm von Aschenhütte nicht bemerkbar. Das Ausmaß der Klimaverschlechterung hat offensichtlich die ökologischen Grenzwerte der damals im Gebiet vorhandenen Pflanzenarten nicht überschritten. Solifluktion und terrestrischer Sedimenteintrag in den Uferbereich des Sees lassen das ausgehende Brörup-Interstadial, das folgende Rederstall-Stadial und das Stillfried-BInterstadial als morphodynamisch besonders aktive Phasen der Weichsel-Kaltzeit erscheinen. Die pollenanalytischen Datierungen bestätigen die grundsätzliche Richtigkeit der von SCHÖNHALS et al. (1964), ROHDENBURG et al. (1966) und RICKEN (1983) vorgenommenen zeitlichen Einstufung der Lößpaläoböden. Bedingt durch einen unterschiedlichen Zersetzungsgrad der organischen Substanz - er ist in den Torfen geringer als in den limnischen Sedimenten - unterscheiden sich die Gesamtkohlenstoffgehalte und die $\mathrm{C} / \mathrm{N}-$ Verhältnisse in den einzelnen stratigraphischen Abschnitten deutlich voneinander. Die Rekonstruktion der Seespiegeländerungen ergab relative Höchststände unmittelbar am Ende des Eem-Interglazials (frühes Herning-Stadial) und im Brörup-Interstadial sowie einen Tiefstand vor Beginn des Brörup-Interstadials (spätes Herning-Stadial).

\section{Dank}

Die Verfasser danken E. BRUNOTTE (Köln), K. GARLEFF (Bamberg) und H. WEINBERG (Hamburg) für Hilfe bei den Geländearbeiten, W. WILLE (Tübingen) für erste Pollenanalysen, B. MEYER (Göttingen) für die Ermöglichung der Kohlenstoff- und Stickstoffbestimmungen und U. JASCHINSKI (Göttingen) für das Zeichnen der Pollendiagramme.

\section{Schriftenverzeichnis}

Aalto, M. (1970): Potamogetonaceae fruits. I. Recent and subfossil endocarps of the fennoscandian species. Acta Bot. Fenn., 88: 1-85; 194 Abb.; Helsinki.

ANDERSEN, S. TH. (1961): Vegetation and its environment in Denmark in the Early Weichselian Glacial. - Danmarks Geol. Unders., II. R. No. 75: 9-175, 33 Tab., 15 Taf.; Kфbenhavn.

ANDERSEN, S. TH. (1980): Early and Late Weichselian chronology and birch assemblages in Denmark. - Boreas, 9: 53-69, 16 Abb., 6 Tab.; Oslo.

AverdieCK, F.-R. (1967): Die Vegetationsentwicklung des Eem-Interglazials und der Frühwürm-Interstadiale von Odderade/Schleswig Holstein. - Fundamenta, Monographien zur Urgeschichte, B/2: 101-105, 3 Abb., 2 Tab., 4 Taf.; Köln.

Behre, K.-E. (1962): Pollen- und diatomeenanalytische Untersuchungen an letztinterglazialen Kieselgurlagern der Lüneburger Heide. - Flora, 152: 325-370, 8 Abb., 3 Taf.; Jena.

- (1974): Die Vegetation im Spätpleistozän von Osterwanna/Niedersachsen. - Geol. Jb., A 18: 3-48, $8 \mathrm{Abb} ., 5$ Taf.; Hannover.

- \& Lade, U. (1986): Eine Folge von Eem und 4 Weichsel-Interstadialen in Oerel/Niedersachsen und ihr Vegetationsablauf. - Eiszeitalter und Gegenwart, 36: 11-36, 12 Abb., 2 Tab., 2 Taf.; Hannover.

CEPEK, A. G., ERD, K. \& ZWIRNER, R. (1981): Drei Interglaziale in einer mittel- bis jungpleistozänen Schichtenfolge östlich von Berlin. - Z. Angewandte Geol., 27: 397-405, 3 Abb., 4 Tab.; Berlin.

DuPHORN, K. (1974): Norddeutschland und angrenzende Gebiete im Eiszeitalter. - 500 S., 91 Abb., 26 Tab.; Stuttgart (Koehler).

ERD, K. (1970): Pollen-analytical classification of the Middle Pleistocene in the German Democratic Republic. - Palaeogeogr., Palaeoclimatol., Palaeoecol., 8: 129-145, 7 Abb.; Amsterdam.

- (1973): Pollenanalytische Gliederung des Pleistozäns der Deutschen Demokratischen Republik. - Z. geol. Wiss. Berlin, 1: 1087-1103, 8 Abb., 1 Tab.; Berlin.

Freising, H. \& FILZER, P. (1978): Ein pollenführendes Interglazialvorkommen aus dem Neckarbecken bei Leutenbach, Lkr. Waiblingen. - Jh. Ges. Naturkde Württemberg, 133: 88-107, 1 Abb., 3 Tab.; Stuttgart.

GRÜGER, E. (1968): Vegetationsgeschichtliche Untersuchungen an cromerzeitlichen Ablagerungen im nördlichen Randgebiet der deutschen Mittelgebirge. - Eiszeitalter und Gegenwart, 18: 204-235, 3 Abb., 5 Taf.; Öhringen.

HaAse, G., Lieberoth, J. \& Ruske, R. (1970): Sedimente und Paläoböden im Lößgebiet. - Peterm. geogr. Mitt., Erg. H. 274: 99-210, 57 Abb.; Gotha. 
HALLIK, R. (1953): Zur Feinstratigraphie des Eem-Interglazials. - Geol. Jb., 68: 179-184, 1 Abb.; Hannover.

JORDAN, H. (1976): Die Terrassenkiese im Sösetal am Harz. - Geol. Jb., A 36: 75-101, 13 Abb., 1 Tab.; Hannover.

Jung, W., Beug, H.-J. \& DeHM, R. (1972): Das Riß/WürmInterglazial von Zeifen, Landkreis Laufen a. d. Salzach. - Bayer. Akad. Wiss., Math.-Nat. Kl., Abh. N. F. 151: 1-131, 15 Abb., 7 Taf.; München.

Mania, D. \& Stechemesser, H. (1970): Jungpleistozäne Klimazyklen im Harzvorland. - Peterm. geogr. Mitt., Erg. H. 274: 39-55, 3 Abb., 1 Tab.; Gotha.

MENKE, B. (1968): Beiträge zur Biostratigraphie des Mittelpleistozäns in Norddeutschland (pollenanalytische Untersuchungen aus Westholstein). - Meyniana, 18: 35-42, 6 Abb., Kiel.

MenKe, B. (1976): Neue Ergebnisse zur Stratigraphie und Landschaftsentwicklung im Jungpleistozän Westholsteins. - Eiszeitalter und Gegenwart, 27: 53-68, 1 Abb., 3 Tab.; Öhringen.

- \& TYNNI, R. (1984): Das Eeminterglazial und das Weichselfrühglazial von Rederstall/Dithmarschen und ihre Bedeutung für die mitteleuropäische Jungpleistozän-Gliederung. - Geol. Jb., A 76: 3-120, 18 Abb., 7 Tab., 9 Taf.; Hannover.

Merkt, J., LÜttig, G. \& Schneekioth, H. (1971): Vorschlag zur Gliederung und Definition der limnischen Sedimente. - Geol. Jb., 89: 607-623, 1 Taf.; Hannover.

MÜLLER, H. (1965): Eine pollenanalytische Neubearbeitung des Interglazial-Profils von Bilshausen (Unter-Eichsfeld). — Geol. Jb., 83: 327-352, 5 Tab., 2 Taf.; Hannover.

- (1974): Pollenanalytische Untersuchungen und Jahresschichtenzählungen an der eem-zeitlichen Kieselgur von Bispingen/Luhe. - Geol. Jb., A 21: 149-169, 5 Abb., 3 Tab., Hannover.

Naucke, W. (1980): Chemie von Moor und Torf. - In: GÖTtLich, K. (Hsgb.): Moor- und Torfkunde: 270 S., 135 Abb., 22 Tab.; Stuttgart (Schweizerbart).

RABIEN, I. (1953): Die Vegetationsentwicklung des Interglazials von Wallensen in der Hilsmulde. - Eiszeitalter und Gegenwart, 3: 96-127, 11 Abb., 1 Tab.; Öhringen.

RICKEN, W. (1982): Quartäre Klimaphasen und Subrosion als Faktoren der Bildung von Kies-Terrassen im südwestlichen Harzvorland. - Eiszeitalter und Gegenwart, 32: 109-136, 9 Abb., 3 Tab., 1 Taf.; Hannover.
RICKEN, W. (1983): Mittel- und jungpleistozäne Lößdecken im südwestlichen Harzvorland. - In: BORK, H.-R. \& RICKEN, W. (Hsgb): Bodenerosion, holozäne und pleistozäne Bodenentwicklung. - Catena, Supplement 3: 95-138, 12 Abb., 2 Tab.; Braunschweig.

RICKEN, W. \& MEYER, B. (1982): Lößbürtige Böden des Jungquartärs zwischen Harz und Leine. - Mitt. dtsch. bodenkdl. Ges., 34: 249-268, 6 Abb.; Göttingen.

Rohdenburg, H. \& MeYer, B. (1966): Zur Feinstratigraphie und Paläopedologie des Jungpleistozäns nach Untersuchungen an südniedersächsischen und nordhessischen Lößprofilen. - Mitt. dtsch. bodenkdl. Ges., 5: 1-137, 25 Abb.; Göttingen.

Schachtschabel, P., Blume, H.-P., Hartge, K. H. \& SCHWERTMANN, U. (1976): Lehrbuch der Bodenkunde. — 394 S., 153 Abb., 77 Tab., 1 Taf.; Stuttgart (Enke).

SCHNEEKIOTH, H. (1966): Ergebnisse weiterer Untersuchungen an den interstadialen Ablagerungen der WeichselEiszeit in Oerel/Krs. Bremervörde. - Z. dtsch. geol. Ges., 116: 773-796, 6 Abb., 2 Tab.; Hannover.

Schönhals, E., Rohdenburg, H. \& Semmel, A. (1964): Ergebnisse neuer Untersuchungen zur Würmgliederung in Hessen. - Eiszeitalter und Gegenwart, 15: 199-206, 1 Abb.; Öhringen.

STREmme, H. E. \& MenKe, B. (1980): Quartär-Exkursionen in Schleswig-Holstein. - Seventh session of the International Geological Correlation Programme, project 24: Quaternary glaciations in the northern hemisphere: 132 pp., 39 Abb., 7 Tab., 4 Taf.; Kiel.

TURNER, CH. (1975): The correlation and duration of Middle Pleistocene interglacial periods in northwest Europe. - In: ButZer, K. \& IsAAC, G. L. (eds.): After the australopithecines: 911 pp., The Hague.

USINGER, H. (1981): Ein weit verbreiteter Hiatus in spätglazialen Seesedimenten: Mögliche Ursache für Fehlinterpretation von Pollendiagrammen und Hinweis auf klimatisch verursachte Seespiegelbewegungen. - Eiszeitalter und Gegenwart, 31: 91-107, 5 Abb.; Hannover.

ZAGWIJN, W. H. (1961): Vegetation, climate and radiocarbon datings in the late pleistocene of the Netherlands. - Meded. Geol. Stichting, N.S. 14: 15-45, 14 Abb., 2 Tab., 11 Taf.; Maastricht.

Manuskript eingegangen am 15. 10. 1987. 